\title{
MANIPULATOR TRAJECTORIES DURING ORBITAL SERVICING MISSION: NUMERICAL SIMULATIONS AND EXPERIMENTS ON MICROGRAVITY SIMULATOR
}

\author{
T. Rybus and K. Seweryn \\ Space Research Centre (CBK PAN) \\ 18a Bartycka Str., Warsaw 00-716, Poland
}

\begin{abstract}
It is considered to use a manipulator-equipped satellite for performing On-Orbit Servicing (OOS) or Active Debris Removal (ADR) missions. In this paper, several possible approaches are reviewed for end-effector (EE) trajectory planning in the Cartesian space, such as application of the Bézier curves for singularity avoidance and method for trajectory optimization. The results of numerical simulations for a satellite equipped with a 7 degree-of-freedom (DoF) manipulator and results of experiments performed on a planar air-bearing microgravity simulator for a simplified two-dimensional (2D) case with a 2-DoF manipulator are presented. Differences between the free-floating case and the case where Attitude and Orbit Control Systems (AOCS) keep constant position and orientation of the satellite are also shown.
\end{abstract}

\section{INTRODUCTION}

An autonomous satellite equipped with a manipulator could be used for precise capture of a malfunctioned satellite in order to perform repairs, replace certain broken components, or replenish depleted fuel. On-Orbit Servicing could be economically feasible, as it could prolong operational lifetimes of commercial satellites [1]. Moreover, manipulator-equipped satellite could also be used for ADR. Space debris, such as spent rocket stages, defunct satellites, and collisional fragments, threaten existing satellites on low Earth orbit (LEO). A collision of an operational satellite even with a small space debris is disastrous as relative velocity of colliding objects is high. On-orbit debris population in LEO is likely to increase due to collisions between existing debris [2]. Thus, missions are proposed for capturing and removing selected large space debris from orbit (see, e. g., [3]).

In this paper, the focus is on the subject of trajectory planning for a manipulator mounted on a satellite. Planning EE trajectory for safe grasping of

(C) The Authors, published by EDP Sciences. This is an open access article distributed under the terms of the Creative Commons Attribution License 4.0 (http://creativecommons.org/licenses/by/4.0/). 
a selected fixture on the target object is a challenging task. As motions of the manipulator induce reaction torques and forces acting on its base, AOCS of the manipulator-equipped satellite must either be able to keep constant position and orientation of the satellite relative to the target object by fully compensating these reactions, or switched off during the capture manoeuvre. In the second case, the free-floating nature [4] of the satellite-manipulator system must be taken into account during the manipulator EE trajectory planning. Moreover, the EE trajectory must prevent potential collisions of the manipulator with elements of the target object and should avoid singularities of the manipulator (in case of the free-floating system, dynamic singularities must be considered [5]).

Several possible approaches for EE trajectory planning are reviwed, such as application of the Bézier curves for computationally inexpensive planning of trajectories, which can be used to avoid singularities, and a trajectory optimization method that allows minimization of a quadratic norm connected with the power use of motors in manipulator joints. Realization of trajectories is demonstrated in experiments performed on planar air-bearing microgravity simulator (where a satellite model has a 2-DoF manipulator and its motions are limited to two dimensions) and using numerical simulation tool for space robotics (where the satellite has a 7-DoF manipulator). The numerical simulation tool and the airbearing test bed allow for analysis of various trajectories and validation of the satellite-manipulator control system responsible for trajectory planning.

The paper is organized as follows. In section 2, equations describing dynamics of a satellite-manipulator are presented. In section 3, trajectory planning methods are reviewed while in section 4 , planar air-bearing microgravity simulator and simulation tool for space robotics are described. Results of experiments and numerical simulations are presented in section 5 and discussed in section 6 . The paper concludes with a short summary in section 7 .

\section{DYNAMICS OF THE SATELLITE-MANIPULATOR SYSTEM}

In this section, equations for the general case of a spatial $n$-DoF manipulator mounted on a satellite are derived. Coordinate systems and geometrical parameters of the considered satellite-manipulator system are shown in Fig. 1. All equations presented herein are expressed in the inertial reference frame $\mathrm{CS}_{\text {ine }}$.

This section is based on the approach presented in [6]. The position of the manipulator EE can be expressed as

$$
\mathbf{r}_{\mathrm{ee}}=\mathbf{r}_{s}+\mathbf{r}_{q}+\sum_{i=1}^{n} \mathbf{l}_{i}
$$




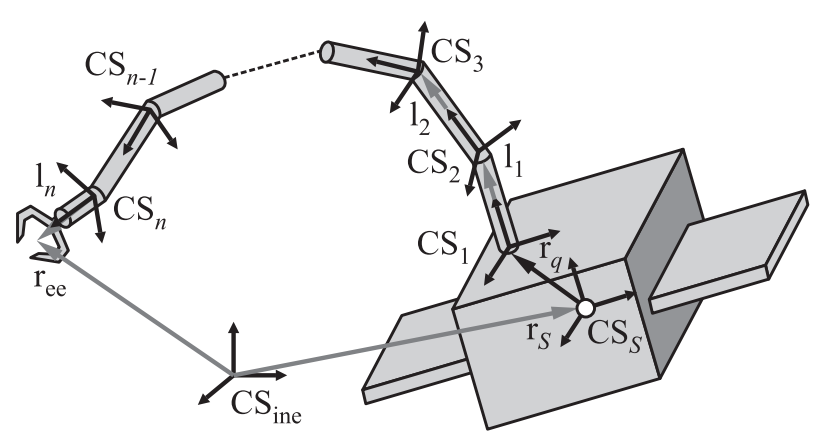

Figure 1 Schematic view of the satellite-manipulator system

where $\mathbf{r}_{s}$ denotes the position of the satellite mass center; $\mathbf{r}_{q}$ is the position of the first kinematic pair of the manipulator with respect to the satellite; and $\mathbf{l}_{i}$ is the position of the $i+1$ kinematic pair in respect to the $i$ th kinematic pair. Linear and angular velocity of the EE can be expressed as

$$
\begin{aligned}
& \mathbf{v}_{\mathrm{ee}}=\mathbf{v}_{s}+\boldsymbol{\omega}_{s} \times\left(\mathbf{r}_{\mathrm{ee}}-\mathbf{r}_{s}\right)+\sum_{i=1}^{n}\left[\mathbf{k}_{i} \times\left(\mathbf{r}_{\mathrm{ee}}-\mathbf{r}_{i}\right)\right] \dot{\theta}_{i} ; \\
& \boldsymbol{\omega}_{\mathrm{ee}}=\boldsymbol{\omega}_{s}+\sum_{i=1}^{n} \mathbf{k}_{i} \dot{\theta}_{i}
\end{aligned}
$$

where $\mathbf{v}_{s}$ and $\boldsymbol{\omega}_{s}$ are the linear and angular velocities of the servicing satellite, respectively; $\mathbf{r}_{i}$ is the position of the $i$ th kinematic pair (in respect to the center of the inertial reference frame $\mathrm{CS}_{\text {ine }}$ ); $\mathbf{k}_{i}$ is the angular velocity vector of the $i$ th kinematic pair; and $\dot{\theta}_{i}$ is the angular velocity of the $i$ th rotational joint. End-effector velocity is expressed in the vector notation:

$$
\left[\begin{array}{c}
\mathbf{v}_{\mathrm{ee}} \\
\boldsymbol{\omega}_{\mathrm{ee}}
\end{array}\right]=\mathbf{J}_{s}\left[\begin{array}{c}
\mathbf{v}_{s} \\
\boldsymbol{\omega}_{s}
\end{array}\right]+\mathbf{J}_{M} \dot{\boldsymbol{\theta}}
$$

where $\mathbf{J}_{s}$ is the Jacobian of the satellite $(6 \times 6$-dimensional matrix $) ; \mathbf{J}_{M}$ is the standard Jacobian of a nonspace manipulator $(6 \times n$-dimensional matrix $)$, while $\dot{\boldsymbol{\theta}}$ is the $n$-dimensional vector containing angular velocities of the manipulator joints. Jacobians $\mathbf{J}_{S}$ and $\mathbf{J}_{M}$ are given by

$$
\begin{aligned}
\mathbf{J}_{s} & =\left[\begin{array}{cc}
\mathbf{I} & \tilde{\mathbf{r}}_{\mathrm{ee} \_s}^{\mathrm{T}} \\
\mathbf{0} & \mathbf{I}
\end{array}\right] ; \\
\mathbf{J}_{M} & =\left[\begin{array}{ccc}
\mathbf{k}_{1} \times\left(\mathbf{r}_{\mathrm{ee}}-\mathbf{r}_{1}\right) & \cdots & \mathbf{k}_{n} \times\left(\mathbf{r}_{\mathrm{ee}}-\mathbf{r}_{n}\right) \\
\mathbf{k}_{1} & \cdots & \mathbf{k}_{n}
\end{array}\right]
\end{aligned}
$$


where $\mathbf{r}_{\mathrm{ee}_{-} s}=\mathbf{r}_{\mathrm{ee}}-\mathbf{r}_{s} ; \mathbf{I}$ is the identity matrix; $\mathbf{0}$ is the zero matrix; and symbol ${ }^{\sim}$ denotes a matrix which is an equivalent of a vector cross product. The kinetic energy of the system is:

$$
\mathbf{T}=\frac{1}{2}\left[\begin{array}{c}
\mathbf{v}_{s} \\
\boldsymbol{\omega}_{s} \\
\dot{\boldsymbol{\theta}}
\end{array}\right]^{\mathrm{T}}\left[\begin{array}{ccc}
\mathbf{A} & \mathbf{B} & \mathbf{D} \\
\mathbf{B}^{\mathrm{T}} & \mathbf{E} & \mathbf{F} \\
\mathbf{D}^{\mathrm{T}} & \mathbf{F}^{\mathrm{T}} & \mathbf{N}
\end{array}\right]\left[\begin{array}{c}
\mathbf{v}_{s} \\
\boldsymbol{\omega}_{s} \\
\dot{\boldsymbol{\theta}}
\end{array}\right]
$$

where the submatrices $\mathbf{A}, \mathbf{B}, \mathbf{D}, \mathbf{E}, \mathbf{F}$, and $\mathbf{N}$ are defined as

$$
\begin{aligned}
& \mathbf{A}=\left(m_{s}+\sum_{i=1}^{n} m_{i}\right) \mathbf{I} \\
& \mathbf{B}=\left(m_{s}+\sum_{i=1}^{n} m_{i}\right) \tilde{\mathbf{r}}_{s_{-} q} ; \\
& \mathbf{D}=\sum_{i=1}^{n} m_{i} \mathbf{J}_{T i} ; \\
& \mathbf{E}=\mathbf{I}_{s}+\sum_{i=1}^{n}\left(\mathbf{I}_{i}+m_{i} \tilde{\mathbf{r}}_{i_{-} s}^{\mathrm{T}} \tilde{\mathbf{r}}_{i_{-} s}\right) \\
& \mathbf{F}=\sum_{i=1}^{n}\left(\mathbf{I}_{i} \mathbf{J}_{R i}+m_{i} \tilde{\mathbf{r}}_{i_{-} s} \mathbf{J}_{T i}\right) ; \\
& \mathbf{N}=\sum_{i=1}^{n}\left(\mathbf{J}_{R i}^{\mathrm{T}} \mathbf{I}_{i} \mathbf{J}_{R i}+m_{i} \mathbf{J}_{T i}^{\mathrm{T}} \mathbf{J}_{T i}\right)
\end{aligned}
$$

where $\mathbf{r}_{s_{-} q}=\mathbf{r}_{s}-\mathbf{r}_{q} ; \mathbf{r}_{i_{-} s}=\mathbf{r}_{i}-\mathbf{r}_{s} ; m_{s}$ denotes the mass of the satellite, while $\mathbf{I}_{s}$ denotes its inertia matrix; $m_{i}$ denotes the mass of the $i$ th manipulator link, while $\mathbf{I}_{i}$ denotes its inertia matrix; $\mathbf{J}_{T i}$ is the translational component of the manipulator Jacobian (expressed in the inertial reference frame); and $\mathbf{J}_{R i}$ is the rotational component of this Jacobian. The momentum $\mathbf{P}$ and the angular momentum $\mathbf{L}=\mathbf{L}_{0}+\mathbf{r}_{s} \times \mathbf{P}$ of the satellite-manipulator system are given by

$$
\left[\begin{array}{c}
\mathbf{P} \\
\mathbf{L}_{0}+\mathbf{r}_{s} \times \mathbf{P}
\end{array}\right]=\mathbf{H}_{2}\left[\begin{array}{c}
\mathbf{v}_{s} \\
\boldsymbol{\omega}_{s}
\end{array}\right]+\mathbf{H}_{3} \dot{\boldsymbol{\theta}}=\left[\begin{array}{l}
0 \\
0
\end{array}\right]
$$

where $\mathbf{L}_{0}$ denotes the initial angular momentum of the system. Matrices $\mathbf{H}_{2}$ and $\mathbf{H}_{3}$ are defined as

$$
\begin{aligned}
\mathbf{H}_{2} & =\left[\begin{array}{cc}
\mathbf{A} & \mathbf{B} \\
\mathbf{B}^{\mathrm{T}}+\tilde{\mathbf{r}}_{s} \mathbf{A} \mathbf{E}+\tilde{\mathbf{r}}_{s} \mathbf{B}
\end{array}\right] ; \\
\mathbf{H}_{3} & =\left[\begin{array}{c}
\mathbf{D} \\
\mathbf{F}+\tilde{\mathbf{r}}_{s} \mathbf{D}
\end{array}\right] .
\end{aligned}
$$


For the sake of simplicity, let us assume that the linear momentum and angular momentum are zero. Thus, let us assume that there are no external torques and forces acting on the system and that at the initial time, both the linear and angular velocities of the satellite-manipulator system are equal to zero in the inertial reference frame. However, as presented in [6], Eq. (1) can be modified to take into account nonzero momentum and angular momentum that, in general, can change due to the actions of external forces and torques (resulting, e.g., from the satellite thrusters or from the gravity gradient). The EE velocity vector is expressed as

$$
\left[\begin{array}{c}
\mathbf{v}_{\mathrm{ee}} \\
\boldsymbol{\omega}_{\mathrm{ee}}
\end{array}\right]=\left(\mathbf{J}_{M}-\mathbf{J}_{s} \mathbf{H}_{2}^{-1} \mathbf{H}_{3}\right) \dot{\boldsymbol{\theta}} .
$$

Relation between the EE velocity and the angular velocities of the kinematic pairs is given by

$$
\dot{\boldsymbol{\theta}}=\left(\mathbf{J}_{M}-\mathbf{J}_{s} \mathbf{H}_{2}^{-1} \mathbf{H}_{3}\right)^{-1}\left[\begin{array}{c}
\mathbf{v}_{\mathrm{ee}} \\
\boldsymbol{\omega}_{\mathrm{ee}}
\end{array}\right],
$$

while the manipulator-equipped satellite velocity is expressed by

$$
\left[\begin{array}{c}
\mathbf{v}_{s} \\
\boldsymbol{\omega}_{s}
\end{array}\right]=-\mathbf{H}_{2}^{-1} \mathbf{H}_{3} \dot{\boldsymbol{\theta}}
$$

The matrix in Eq. (2) can be inverted if the manipulator has 6 joints (minimal number of joints required for realization of a desired EE position and orientation). When $n \neq 6$, Moore-Penrose pseudoinverse of matrix is used.

Angular velocity of the servicing satellite, $\boldsymbol{\omega}_{s}$, determines, through kinematic equations, the transformation matrix between the inertial reference frame and the body-fixed coordinate system. In this paper, Langrangian formalism is used to derive dynamics equations for the considered system. Potential energy is neglected. Generalized coordinates take the following form:

$$
\mathbf{q}_{p}=\left[\mathbf{r}_{s} \boldsymbol{\Theta}_{s} \boldsymbol{\theta}\right]^{\mathrm{T}}
$$

where $\boldsymbol{\Theta}_{s}$ is the orientation of the servicing satellite (Euler angles in $X Y Z$ convention). The general form of Lagrange equation is

$$
\frac{d}{d t}\left(\frac{\partial \mathbf{T}}{\partial \dot{\mathbf{q}}}\right)-\frac{\partial \mathbf{T}}{\partial \mathbf{q}}=\mathbf{Q} .
$$

Here, $\mathbf{Q}$ is the vector of generalized forces:

$$
\mathbf{Q}=\left[\begin{array}{lll}
0 & 0 & \mathbf{u}
\end{array}\right]^{\mathrm{T}}
$$

where $\mathbf{u}$ is the control vector composed of driving torques in manipulator joints. From Eq. (4), generalized equations of motion are derived:

$$
\mathbf{Q}=\mathbf{M}\left(\mathbf{q}_{p}\right) \ddot{\mathbf{q}}_{p}+\mathbf{C}\left(\dot{\mathbf{q}}_{p}, \mathbf{q}_{p}\right) \dot{\mathbf{q}}_{p}
$$


where $\mathbf{M}$ denotes the mass matrix and $\mathbf{C}$ denotes the Coriolis Matrix. No potential forces are included in Eq. (5) as the orbiting system is in the state of free fall. The mass matrix, $\mathbf{M}$, can be expressed as

$$
\mathbf{M}\left(\mathbf{q}_{p}\right)=\left[\begin{array}{ccc}
\mathbf{A} & \mathbf{B} & \mathbf{D} \\
\mathbf{B}^{\mathrm{T}} & \mathbf{E} & \mathbf{F} \\
\mathbf{D}^{\mathrm{T}} & \mathbf{F}^{\mathrm{T}} & \mathbf{N}
\end{array}\right],
$$

while components of the Coriolis matrix, $\mathbf{C}$, are equal to

$$
C_{i j}=\sum_{k=1}^{n}\left(\frac{d m_{i j}}{d q_{k}}-\frac{1}{2} \frac{d m_{j k}}{d q_{i}}\right)
$$

where $m_{i j} \in \mathbf{M}\left(\mathbf{q}_{p}\right)$ and $i, j, k=1, \ldots, n$.

The equations presented above are applicable for a free-floating satellitemanipulator system with zero momentum and angular momentum. If the capture maneuvre is performed with active AOCS (which compensates all reaction forces and torques acting on the satellite and keeps constant position and orientation of the satellite), the trajectory of the manipulator must be planned with the assumption that the manipulator base is fixed. In such a case, equations presented in this section are simplified to equations describing standard nonspace manipulator (such equations can be found, e.g., in [7]).

\section{MANIPULATOR TRAJECTORIES}

\subsection{Trajectory Planning Stage}

In operations of a manipulator mounted on a satellite, let us distinguish two stages: $(i)$ manipulator trajectory planning stage; and $(i i)$ trajectory realization stage. During the capture maneuver, trajectory planning is performed while the manipulator-equipped satellite is waiting in a safe point. Realization of the planned trajectory requires controller that works in a real time during the manipulator motion. This paper focuses on the trajectory planning stage. The purpose of this stage is to find a suitable trajectory of the manipulator from a specified initial state of the system to a desired final state. Trajectory can be planned in the configuration space (positions of manipulator joints) or in the Cartesian coordinates (EE positions) using the methods described in this section. The inverse dynamics problem is solved at this stage: trajectory given in the EE velocity space is transformed to joint velocities using inverse of the body dynamic Jacobian. Then, reference control torques in the manipulator joints required for realization of this trajectory are computed with Eq. (5). Reference control torques can be used by the control system during realization of the planned trajectory. 


\subsection{Optimal Trajectories}

Optimization plays an important role in manipulator trajectory planning. It can allow minimization of the manipulator influence on the satellite position and orientation or it can be used to minimize control torques applied in manipulator joints. The approach for the EE trajectory optimization presented herein is based on [6]. We are seeking such EE trajectory from an initial state to a desired final state that will be characterized by the minimal power use of motors driving the manipulator joints. Optimized functional $G$ has the following form:

$$
G\left(\mathbf{q}_{v p}(t), \mathbf{u}(t), t\right)=\int_{t_{0}}^{t_{f}} L\left(\mathbf{q}_{v p}(t), \mathbf{u}(t), t\right)+\boldsymbol{\lambda}_{v p}(t)^{\mathrm{T}} \mathbf{g}\left(\mathbf{q}_{v p}(t), \mathbf{u}(t), t\right) d t .
$$

Here, $L$ is the cost functional; $\mathbf{q}_{v p}=\left[\begin{array}{ll}\mathbf{q}_{v} & \mathbf{q}_{p}\end{array}\right]^{\mathrm{T}}$ where $\mathbf{q}_{v}$ is the derivative of the generalized coordinates $\mathbf{q}_{p}$ in respect to time; and $\boldsymbol{\lambda}_{v p}=\left[\begin{array}{ll}\lambda_{v} & \lambda_{p}\end{array}\right]^{\mathrm{T}}$ where $\lambda_{p}$ and $\lambda_{v}$ denote the Lagrange multipliers associated with $\mathbf{q}_{p}$ and $\mathbf{q}_{v}$, respectively. Direct dynamics of the satellite-manipulator system are described by

$$
\mathbf{g}=\left[\begin{array}{c}
\dot{\mathbf{q}}_{v} \\
\dot{\mathbf{q}}_{p}
\end{array}\right]=\left[\begin{array}{c}
\mathbf{M}^{-1}\left(\mathbf{Q}-\mathbf{C q}_{v}\right. \\
\mathbf{q}_{v}
\end{array}\right]
$$

A quadratic norm connected with the power use of manipulator motors is chosen as a functional to be minimized:

$$
L=\frac{1}{2} \mathbf{u}^{\mathrm{T}} \mathbf{u}
$$

Hamiltonian of the system is given by

$$
H=L+\lambda_{v p}^{\mathrm{T}} \mathbf{g} .
$$

The extremum of $G$ corresponds to the following condition imposed on the Hamiltonian, $H$ :

$$
\frac{\partial H}{\partial \mathbf{u}}=0
$$

Equation (7) is used to find the control vector $\mathbf{u}$. State vector is defined as

$$
\mathbf{x}=\left[\begin{array}{llll}
\mathbf{q}_{v} & \mathbf{q}_{p} & \boldsymbol{\lambda}_{v} & \boldsymbol{\lambda}_{p}
\end{array}\right]^{\mathrm{T}} .
$$

One obtains a set of differential equations that minimizes the functional $G$ and satisfies the boundary conditions:

$$
\left[\begin{array}{c}
\dot{\mathbf{q}}_{v p} \\
\dot{\boldsymbol{\lambda}}_{v p}
\end{array}\right]=\left[\begin{array}{c}
\mathbf{g} \\
-\frac{\partial L}{\partial \mathbf{q}_{v p}}-\boldsymbol{\lambda}_{v p}^{\mathrm{T}} \frac{\partial \mathbf{g}}{\partial \mathbf{q}_{v p}}
\end{array}\right]
$$


A Boundary Value Problem (BVP) is formulated and Eq. (8) is solved with boundary conditions and additional equations, which must be satisfied by the algorithm used to solve the BVP. The boundary conditions determine the initial state of the system, i. e., $\mathbf{q}_{v p}$ at the initial time $t_{0}$ and values of Lagrange multipliers at the final time $t_{f}$ :

$$
\boldsymbol{\lambda}_{v p}\left(t_{f}\right)=\left(\mathbf{v}^{\mathrm{T}} \frac{\partial \boldsymbol{\psi}}{\partial \mathbf{q}_{v p}}\right)_{t=t_{f}}
$$

where function $\boldsymbol{\psi}$ describes the final desired EE position and orientation $(\psi$ $\left.=\left[\begin{array}{rl}\mathbf{r}_{\mathrm{ee}} & \boldsymbol{\Theta}_{\mathrm{ee}}\end{array}\right]^{\mathrm{T}}\right)$. Additional parameters $\mathbf{v}$ are determined by the algorithm to satisfy equations for $\psi$.

\subsection{Trajectories Based on Bézier Curves}

In this section, application of the Bézier curves for trajectory planning (introduced and described in detailes in [8]) is presented. Use of the Bézier curves for trajectory planning is less computationally demanding than trajectory optimization presented in subsection 3.2. Such curves can be used for relatively simple planning of trajectories that avoid singularities. To present the problem of singularities, the relation between the EE velocity and the angular velocities of the kinematic pairs given by Eq. (2) is replaced by

$$
\dot{\boldsymbol{\theta}}=\left(\mathbf{J}_{\mathrm{dyn}}^{b}\right)^{-1}\left[\begin{array}{l}
\mathbf{v}_{\mathrm{ee}} \\
\boldsymbol{\omega}_{\mathrm{ee}}
\end{array}\right]
$$

where $\mathbf{J}_{\text {dyn }}^{b}$ is the body dynamic Jacobian [5] that is dependent on the state of the system (including position of manipulator joints $\boldsymbol{\theta}$ ). Equation (9) becomes singular if, and only if, the variable $\boldsymbol{\theta}$ reaches a point in which the Jacobian is not invertible. Thus, in some configurations of the manipulator, the Jacobian operator loses rank and we are not able to generate any velocity vector in the Cartesian space. To assess how far the manipulator is from a singular configuration for a given position of manipulator joints, let us use a manipulability measure defined as

$$
N_{\mathrm{JJ}}=\operatorname{det}\left[\mathbf{J}_{\text {dyn }}^{b}\left(\mathbf{J}_{\text {dyn }}^{b}\right)^{\mathrm{T}}\right] \text {. }
$$

For the EE trajectory planning in Cartesian coordinates, the algorithm should either be able to avoid singularities or to deal with them (as, e. g., in [9]). In this section, the focus is on application of the Bézier curves for singularity avoidance. Modifications of curve shape allow for avoidance of singularities. The selection of the Bézier curve parameters responsible for its shape is intuitive and simple. In three-dimensional (3D) space, the third-order Bézier curve has the following form:

$$
\mathbf{P}(j)=(1-j)^{3} \mathbf{P}_{b}+3(1-j)^{2} j \mathbf{P}_{1}+3(1-j) j^{2} \mathbf{P}_{2}+j^{3} \mathbf{P}_{e}
$$


where $j \in[0 ; 1]$, and is characterized by four points: the initial EE position $\mathbf{P}_{b}$, the final EE position $\mathbf{P}_{e}$, and two intermediate points $\left(\mathbf{P}_{1}\right.$ and $\left.\mathbf{P}_{2}\right)$. The curve starts at $\mathbf{P}_{b}$ going towards $\mathbf{P}_{1}$ and arrives at $\mathbf{P}_{e}$ coming from the direction of $\mathbf{P}_{2}$. Intermediate points determine shape of the curve but do not lie on the curve. Positions of the intermediate points can be described with respect to the EE initial and final position by parameters such as angles and distances to the points. The selection of parame-

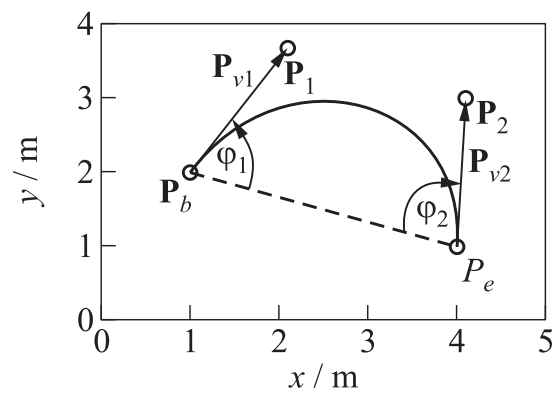

Figure 2 Exemplary Bézier curve in 2D space ters responsible for curve shape can be performed by an automatic algorithm. For the planar (2D) case, an exemplary Bézier curve is presented in Fig. 2.

\subsection{Trajectories for the Capture Manoeuvre}

In the orbital capture manoeuvre, we seek such a trajectory of the manipulator that will bring the satellite-manipulator system from a given initial state to a final state in which the EE position will coincide with a position of the selected grasping point on the target object. As the target object can be tumbling, the trajectory of the gasping point can form a complex curve on the surface of a sphere. Equations describing the motion of the target object grasping point were presented in [10]. Here, the same approach as described in details in [10] is used, where the trajectory planning during the capture manoeuvre is divided into two phases:

(i) motion of the EE from the initial position to an intermediate point; and

(ii) final approach of the EE from the intermediate point to the grasping point position on the target object.

In the second phase, the simplest approach to avoid the risk of collision between the EE or elements of the manipulator and the target object is to select a straightline EE trajectory defined in the coordinate system fixed at the grasping point. In this paper, the focus is on this simple approach but other approaches for trajectory planning during the capture manoeuvre could also be implemented. As the structure of the target object may be complicated (e.g., arrays of solar panels), the number of obstacles in the workspace of the manipulator may be high. In such a case, Rapidly-exploring Random Trees (RRT) algorithm could be used for trajectory planning of the free-floating satellite-manipulator system [11]. 


\section{TOOLS FOR ANALYSIS AND VALIDATION OF TRAJECTORY PLANNING ALGORITHMS}

\subsection{Planar Air-Bearing Microgravity Simulator}

The planar air-bearing microgravity simulator at CBK PAN is a test bed that allows experimental validation of trajectory planning algorithms for free-floating satellite-manipulator systems [12]. Experiments performed on this test bed and presented in this paper served two purposes: $(i)$ to demonstrate the trajectory optimization method described in subsection 3.2 for a real system; and ( $i i)$ to validate numerical simulations performed with the use of simultion tool for space robotics. The main element of the test bed at CBK PAN is a $2 \times 3$-meter granite table, flat and precisely levelled. A model of a satellite-manipulator system is mounted on planar air-bearings that allow almost frictionless motion on the table surface, thus simulating microgravity conditions in two dimensions. Schematic view of the satellite-manipulator system used on this test bed is presented in Fig. 3.

The manipulator has 2 DoF (both joints are rotational). Each joint consists of a direct-current (DC) motor, a harmonic drive, and an absolute optical encoder. Manipulator links are made from aluminum profiles, while the model of the satellite consists of an aluminum plate with a gas canister attached in its center. In this test bed, the satellite is supported by three planar air-bearings that form a three-point stance. Additional air-bearings are used to independently support each manipulator link, thus allowing tests of relatively long and heavy manipulators, which have significant influence on the position and orientation of the satellite model. Resilient suspension plates are used in the manipulator joints

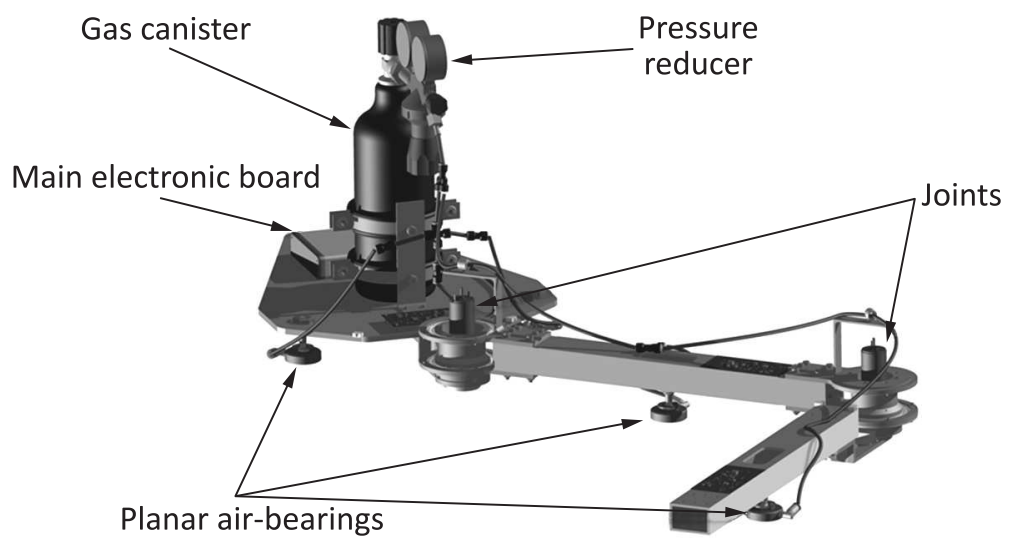

Figure 3 Schematic view of the planar satellite-manipulator system 
Table 1 Geometrical and mass properties of the planar satellite-manipulator system

\begin{tabular}{lcc}
\hline \multicolumn{1}{c}{ Parameter } & Symbol & Value \\
\hline Satellite model mass & $m_{0}$ & $12.9 \mathrm{~kg}$ \\
Satellite model inertia & $I_{0}$ & $0.208 \mathrm{~kg} \cdot \mathrm{m}^{2}$ \\
Link 1 mass & $m_{1}$ & $4.5 \mathrm{~kg}$ \\
Link 1 moment of inertia & $I_{1}$ & $0.32 \mathrm{~kg} \cdot \mathrm{m}^{2}$ \\
Link 1 length & $l_{1}$ & $0.62 \mathrm{~m}$ \\
Link 2 mass & $m_{2}$ & $1.5 \mathrm{~kg}$ \\
Link 2 moment of inertia & $I_{2}$ & $0.049 \mathrm{~kg} \cdot \mathrm{m}^{2}$ \\
Link 2 length & $l_{2}$ & $0.6 \mathrm{~m}$ \\
Mass ratio: $\left(m_{1}+m_{2}\right) / m_{0}$ & $k$ & 0.465 \\
\hline
\end{tabular}

for compensation of possible vertical misalignments between components of the system (all 5 support points must be ideally coplanar in order to allow proper operation of the bearings and free planar motion of the system). Pressurized air is distributed from the gas canister located on the satellite model to all air bearings through flexible hoses (rotational pneumatic connectors are used to transmit air through the manipulator joints). The main electronic board contains onboard computer that is responsible for mode management, control of the manipulator $\mathrm{EE}$ in the Cartesian space, and data collection. Two joint-controller boards are responsible for control of the manipulator DC motors and realization of trajectory. Geometrical and mass properties of the satellite-manipulator system are presented in Table 1. During the experiments, a visual pose estimation system is used to track the satellite-manipulator system. Visual pose estimation provides position and orientation of the EE and of the satellite mock-up.

\subsection{Simulation Tool for Space Robotics}

Simulation tool for space robotics has been under development at CBK PAN since 2009 [13] and is intended for simulations of a manipulator-equipped satellite during the orbital capture manoeuvre, e.g., in ADR or OOS mission. The tool is based on dedicated functions for manipulator trajectory planning created in Matlab and models of the spacecraft and its robotic arm created in SimMechanics (software based on Simscape, the platform product for Matlab Simulink). Three stages can be distinguished in the operations of the simulation tool:

(i) manipulator trajectory planning;

(ii) simulation of system behavior; and

(iii) postprocessing. 


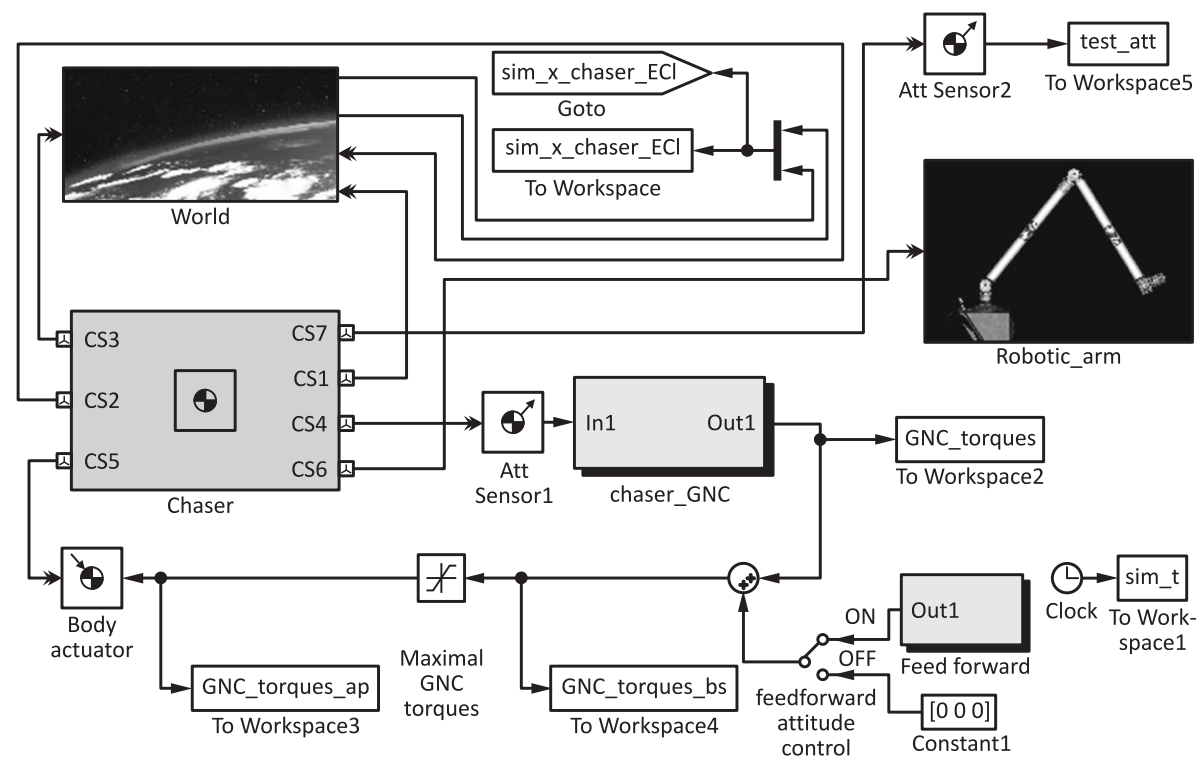

(a)

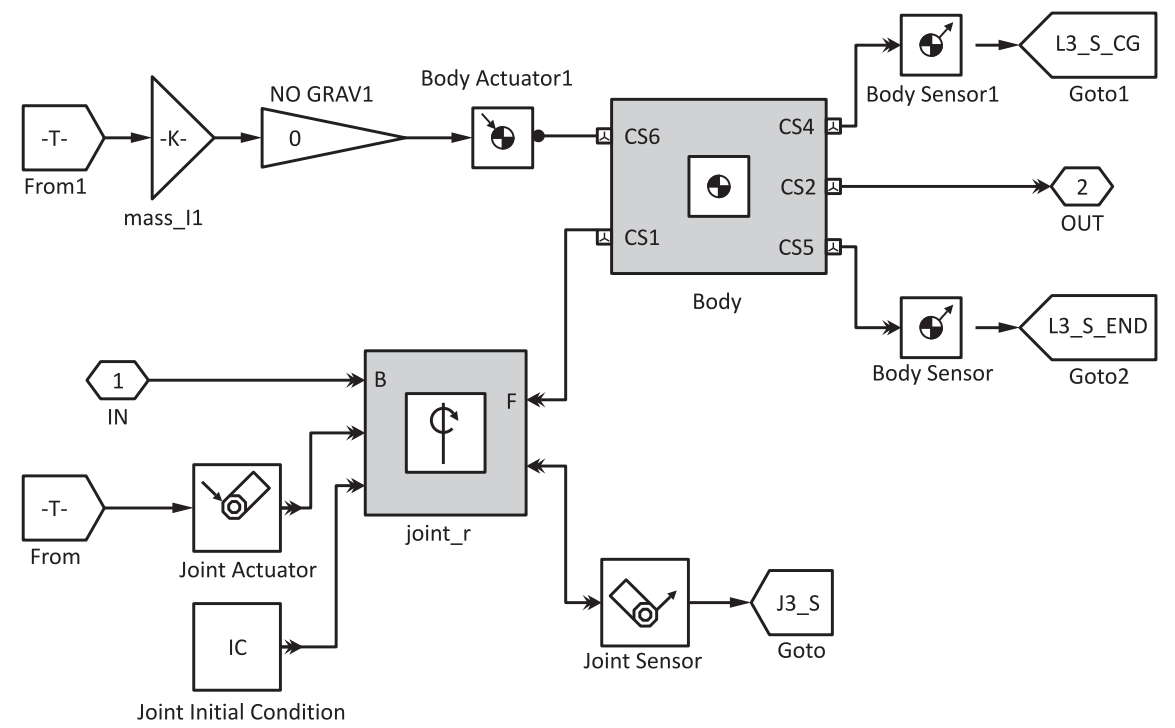

(b)

Figure 4 Simulink model of the satellite-manipulator system $(a)$ and model of manipulator joint $(b)$ 
During trajectory planning stage, the methods presented in section 3 can be used to find a suitable trajectory of the manipulator from a specified initial state of the system to a desired final state. After the trajectory planning phase, the simulation tool is used to perform a numerical simulation of system behavior, i.e., to solve the forward dynamics problem. This stage is performed in Matlab Simulink and based on a model of the system built in SimMechanics. Simscape solver works autonomously and creates the physical network from the block model of the system and then the system of equations for the model is constructed and solved $[14,15]$. In this simulation stage, various disturbances and external forces acting on the system can be added. Control torques for manipulator joints computed during the first stage are applied in appropriate blocks. At this stage, the model of a control system can be used to ensure proper realization of selected trajectory despite disturbances. Postprocessing is the final stage and is initialized after the simulation. In this stage, plots are automatically generated based on the measurements of "virtual" sensors in Simulink. Animation of the satellite-manipulator motion can be displayed.

Overview of the Simulink model of the satellite-manipulator system is presented in Fig. 4a. The satellite is modeled as a rigid body, using a SimMechanics build-in rigid body block. Specific use of a 6 -DoF joint block allows simulation of rotational and translational motion of this satellite. The manipulator is attached to the satellite at a specified mounting point. The satellite is interacting with orbital environment through a "world" block. The manipulator is modeled as a chain of rigid bodies connected by rotational joints. A block containing the manipulator joint is presented in Fig. 4b. Such a joint is built from a "revolute joint" block, a "rigid body" block representing manipulator link and several blocks of "virtual" sensors and actuators. Control torque applied on the joint by "joint actuator" is a sum of control torque computed during trajectory planning and torque coming from the controller that can be operating during the simulation.

Table 2 Geometrical and mass properties of the satellite and 7-DoF manipulator

\begin{tabular}{lccc}
\hline \multicolumn{1}{c}{ Parameter } & Symbol & Value \\
\hline Satellite mass & $m_{s}$ & \multicolumn{3}{c}{$100 \mathrm{~kg}$} \\
& $\mathbf{I}_{s}$ & {$\left[\begin{array}{ccc}2.83 & 0 & 0 \\
0 & 6.08 & 0 \\
0 & 0 & 7.42\end{array}\right] \mathrm{kg} \cdot \mathrm{m}^{2}$} \\
Satellite moment of inertia & & 7 \\
& $n$ & $3.1 \mathrm{~m}$ \\
Number of manipulator DoF & $l_{m}$ & $15.25 \mathrm{~kg}$ \\
Total length of manipulator & $m_{m}$ \\
Total mass of manipulator & \multicolumn{3}{c}{}
\end{tabular}


In simulations performed with the simulation tool described herein, a model of satellite with a 7-DoF manipulator (classical elbow-like architecture) developed at CBK PAN [16] has been used. The properties of this system are presented in Table 2. Simulation tool could also be used for planar case with properties of the system presented in Table 1.

\section{RESULTS}

\subsection{Trajectory Optimization: Planar Experiment}

In this section, the results of the experiment performed on the planar air-bearing microgravity simulator are presented. This experiment served two purposes:
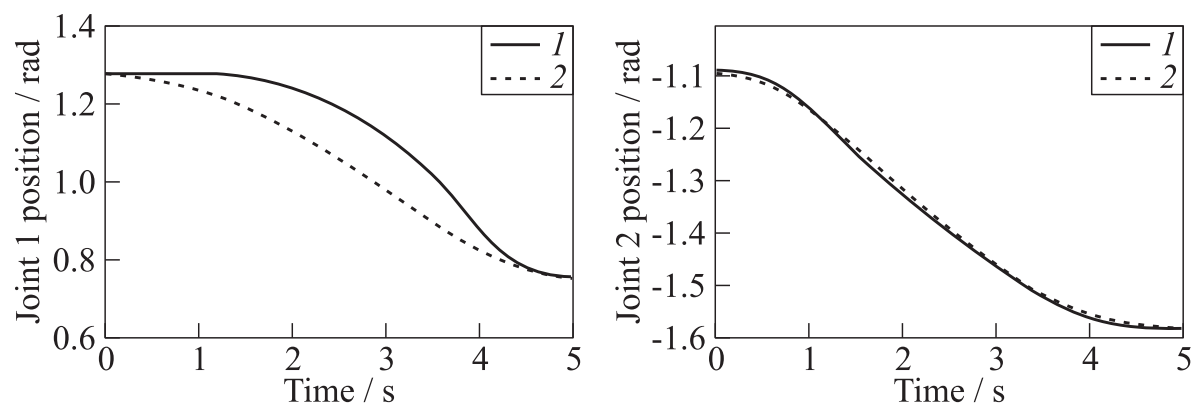

Figure 5 Reference positions of manipulator joints during realization of straightline (1) and optimal (2) trajectories
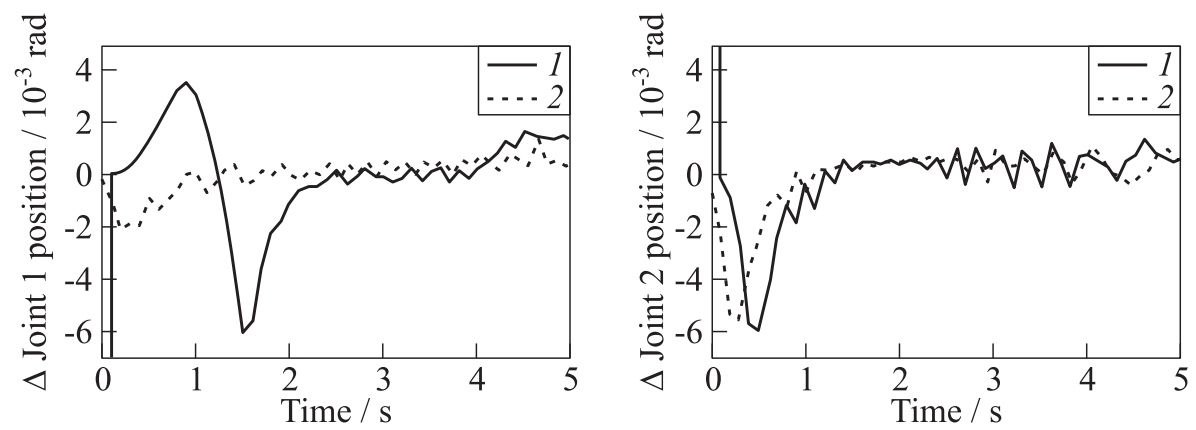

Figure 6 Difference between the reference positions of manipulator joints and positions measured by encoders during realization of straight-line (1) and optimal (2) trajectories 
(i) to demonstrate the trajectory optimization method described in subsection 3.2 for a real system; and (ii) to validate numerical simulations performed with the use of simultion tool for space robotics. The desired final EE position was set $0.3 \mathrm{~m}$ away from the initial position. Two trajectories of the EE were compared: a simple straight line trajectory between the initial and final EE position and a trajectory obtained from the optimization algorithm. Both reference trajectories defined in the Cartesian space were transferred to velocity space and then, using Eqs. (2) and (3), to velocities of manipulator joints. Reference positions

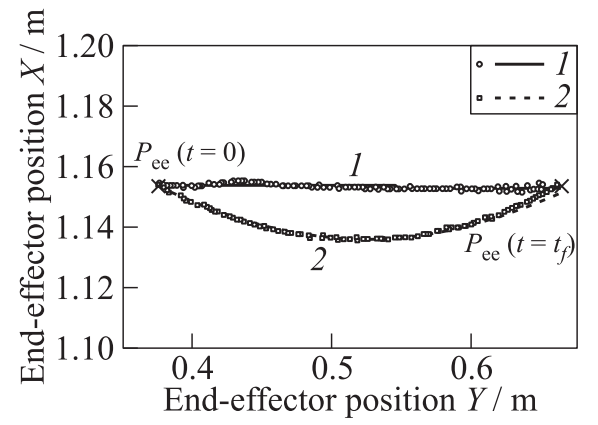

Figure 7 Straight-line (1) and optimal (2) EE reference trajectories (curves) compared with the measurements of EE position obtained during experiment on the planar airbearing microgravity simulator (signs)
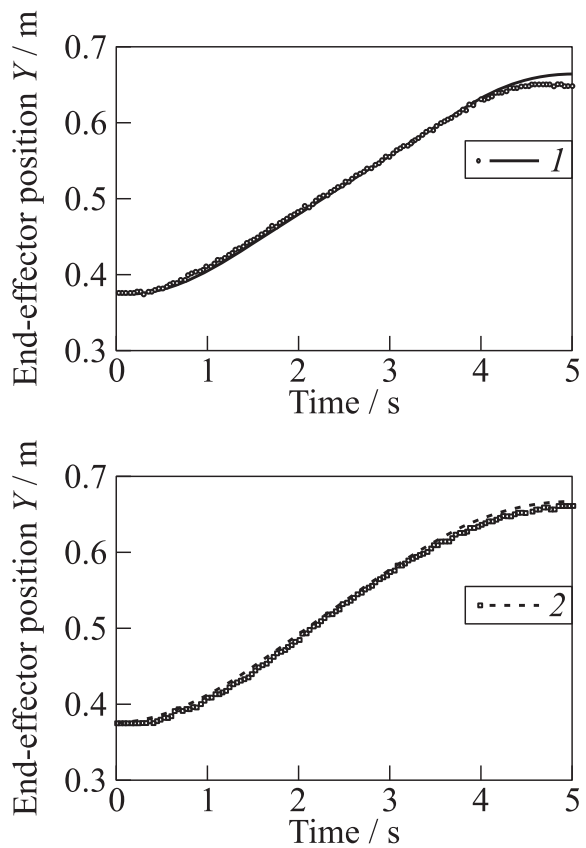

Figure 8 Components of the EE position during realization of straight-line (1) and optimal (2) trajectories: signs - experiment; and curves — reference 


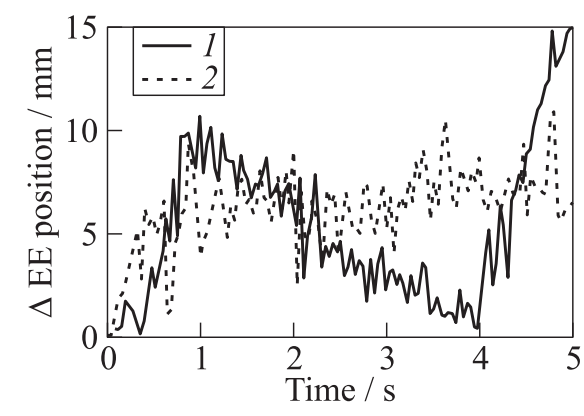

Figure 9 Difference between the reference EE position and EE position measured by the visual pose estimation system during realization of straight-line (1) and optimal (2) trajectories individual components of the EE position during realization of trajectories are shown in Fig. 8. The difference between the reference EE position and EE position measured by the visual pose estimation system is presented in Fig. 9. For both cases, EE trajectory obtained from the experiment is very close to the planned reference trajectory.

Views from the camera of the pose estimation system obtained during realization of the straight-line trajectory are shown in Fig. 10. It can be seen that the
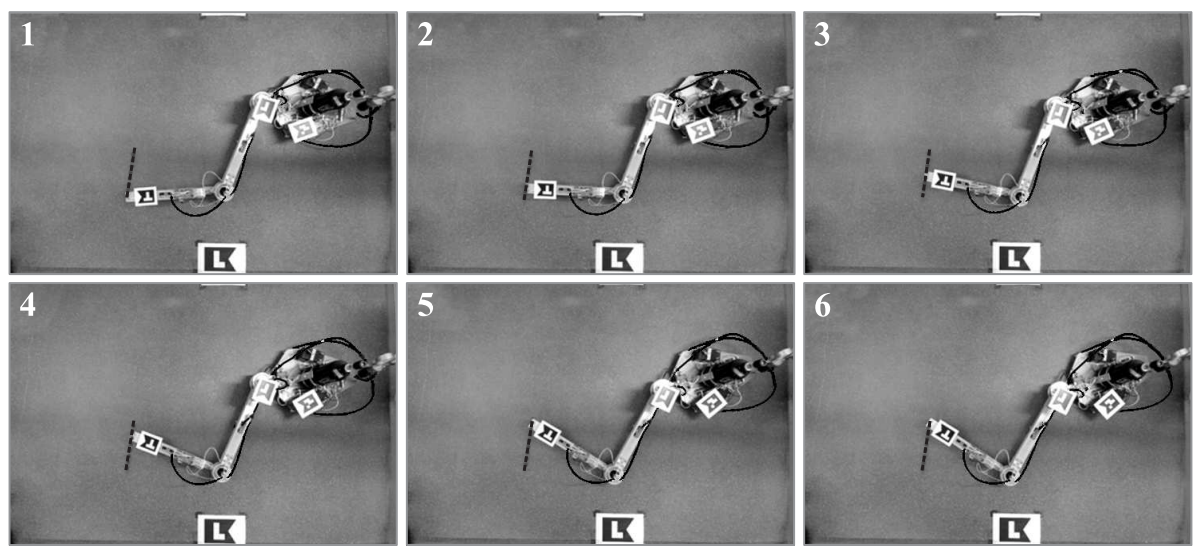

Figure 10 Satellite-manipulator system during realization of straight-line trajectory on the planar air-bearing microgravity simulator (view from the camera used by the pose estimation system) 


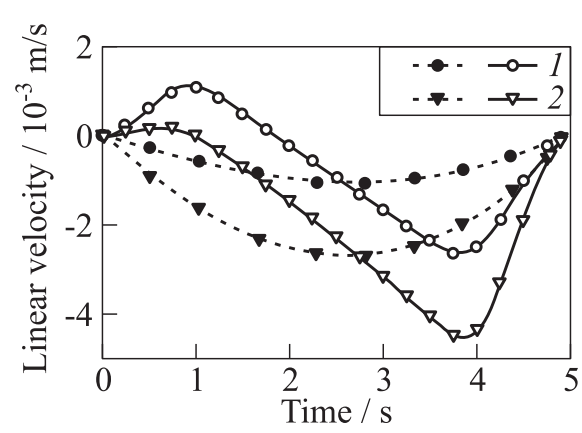

(a)

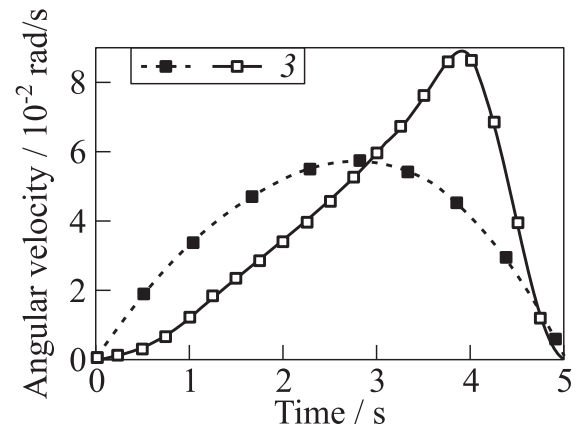

(b)

Figure 11 Reference linear velocity $(a)$ and angular velocity ( $b$ ) of the satellite during realization of straight-line (solid curves) and optimal (dotted curves) trajectories: 1 $V_{x} ; 2-V_{y} ;$ and $3-\omega$

mock-up of satellite is changing its orientation and position due to the reaction forces and reaction torque induced by the motion of the manipulator.

Linear and angular velocity of the manipulator-equipped satellite is presented in Fig. 11. The following values of the cost functional defined in Eq. (6) as a quadratic norm connected with the power use of manipulator motors were obtained: $L_{\text {straight-line }}=0.00524$ for the straight-line trajectory and $L_{\text {optimal }}$ $=0.00261$ for the optimal trajectory. Thus, the optimization procedure presented in subsection 3.2 allowed 50 percent reduction of the cost functional.

\subsection{Trajectories Close to Singularities}

In this subsection, the results of numerical simulations obtained with the simulation tool for space robotics are presented to demonstrate application of the Bézier curves for planning trajectory that can avoid singularities. Some of the results presented herein were previously described in [16].

Map of the manipulability measure in the configuration space (computed with the use of Eq. (10)) is presented in Fig. $12 a$. This map presents the manipulability measure in the entire configuration space, i. e., for all possible positions of manipulator joints 2,3 , and 4 . In the considered manipulator, the position of the first manipulator joint has a very limited influence on this map (because the axis of rotation of this joint is close to the satellite center of mass), while joints 5, 6, and 7 are responsible for the EE orientation and are not taken into account here. Points with lower value of the manipulability are closer to singularities. Points with the manipulability measure higher than 0.014 are not plotted. The presented map of the dynamically singular configurations differs 


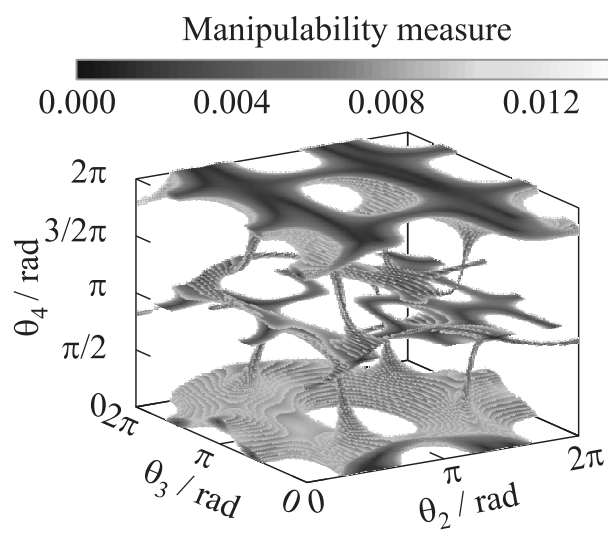

(a)

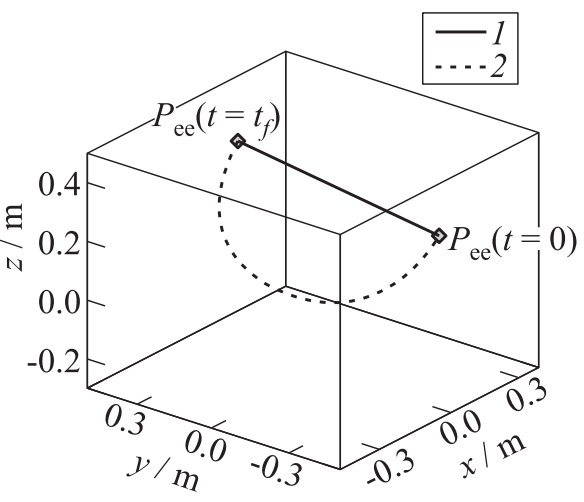

(b)

Figure 12 Map of the manipulability measure for the 7-DoF redundant manipulator with positions of joints 2,3 , and 4 as coordinates $(a)$ and comparison between straightline (1) and Bézier (2) curve trajectories in the Cartesian space (b)
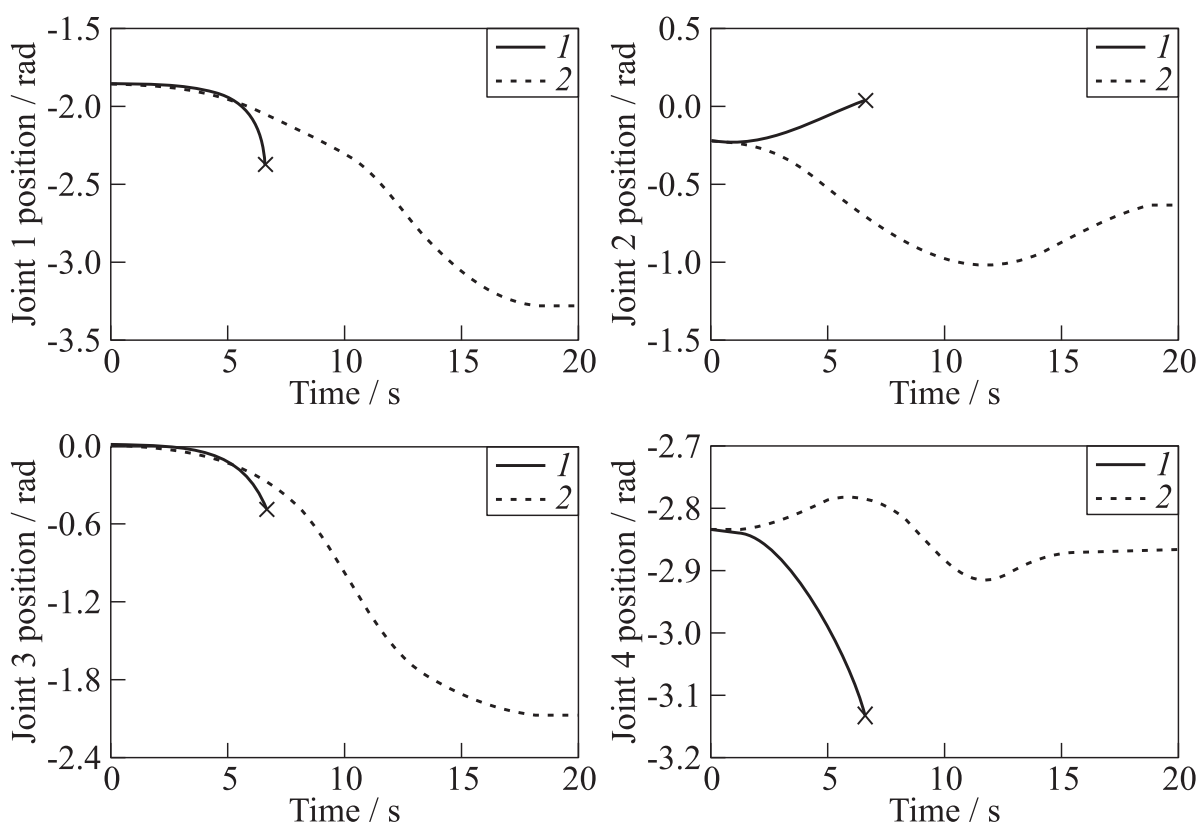

Figure 13 Results of numerical simulations: positions of the first four manipulator joints for straight-line (1) and Bézier curve (2) trajectories (figure adapted from [16]) 
from the map of the kinematic singularities obtained for a fixed-base manipulator.

In Fig. 12b, Bézier curve trajectory is compared with a straightline trajectory from the initial EE position to the desired final EE position.

Positions of manipulator joints that correspond to these trajectories are presented in Fig. 13 where at some point, a configuration close to singular is reached for the straight-line trajectory and body dynamic Jacobian in Eq. (9) can-

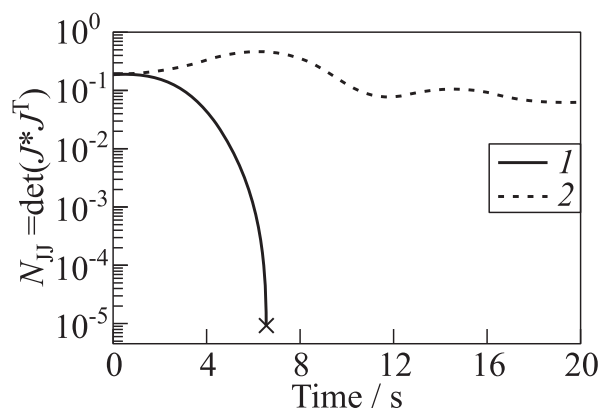

Figure 14 Manipulability measure for straight-line (1) and Bézier curve (2) trajectories not be inverted (straight-line trajectory is only plotted to this point with near-zero manipulability measure). Manipulability measure for both trajectories is presented in Fig. 14. Parameters defining the Bézier curve were obtained by trail-and-error method. The trajectory based on this curve is sufficiently far from any singular configuration and Eq. (9) can be used to compute velocities of manipulator joints for the entire trajectory.

\subsection{Capture Maneuver With and Without Active Altitude and Orbit Control System}

This subsection is devoted to numerical simulations of the capture manoeuvre. Results obtained with the simulation tool for space robotics are presented for two cases:

(i) capture manoeuvre performed without active AOCS (free-floating case); and

(ii) capture manoeuvre performed with a fixed satellite (case corresponding to the satellite with ideal AOCS that compensates all reaction forces and torques to keep constant position and orientation of the manipulatorequipped satellite).

The target object is tumbling. Its initial angular velocity is

$$
\omega=[0.07 \mathrm{rad} / \mathrm{s} 0.018 \mathrm{rad} / \mathrm{s}-0.035 \mathrm{rad} / \mathrm{s}]^{\mathrm{T}} \text {. }
$$

The simple two-phase EE trajectory described briefly in subsection 3.4 and in details in [10] is utilized. This trajectory (shown in Fig. 15) leads from the initial 


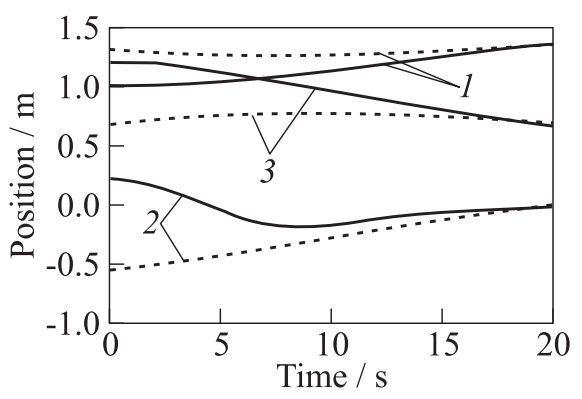

(a)

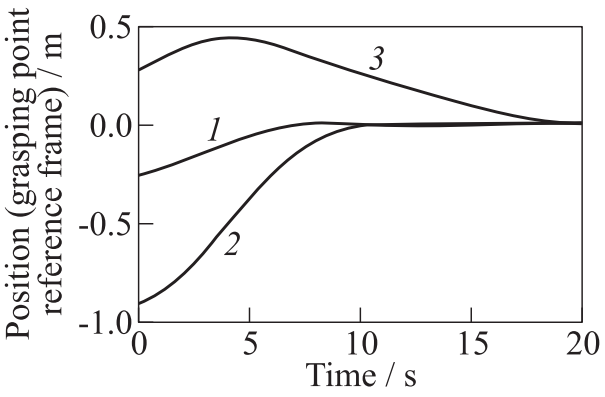

(b)

Figure 15 Positions of the EE (solid curves) and selected grasping point on the target object (dashed curves) in the inertial reference frame $(a)$ and positions of the $\mathrm{EE}$ in the reference frame fixed at the selected grasping point on the target object $(b)$ : $1-X ; 2-Y$; and $3-Z$
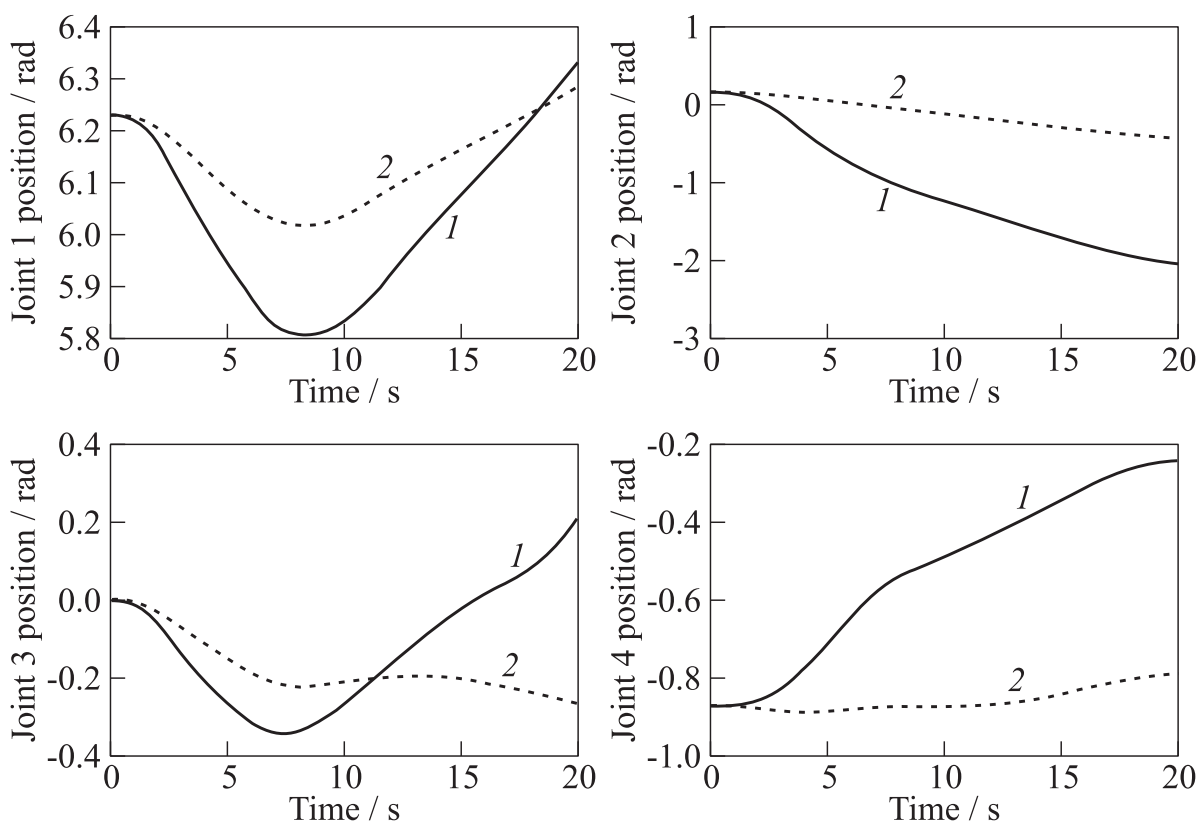

Figure 16 Positions of the first four manipulator joints during the capture manoeuver (comparison between the free-floating (1) and fixed-base (2) cases) 

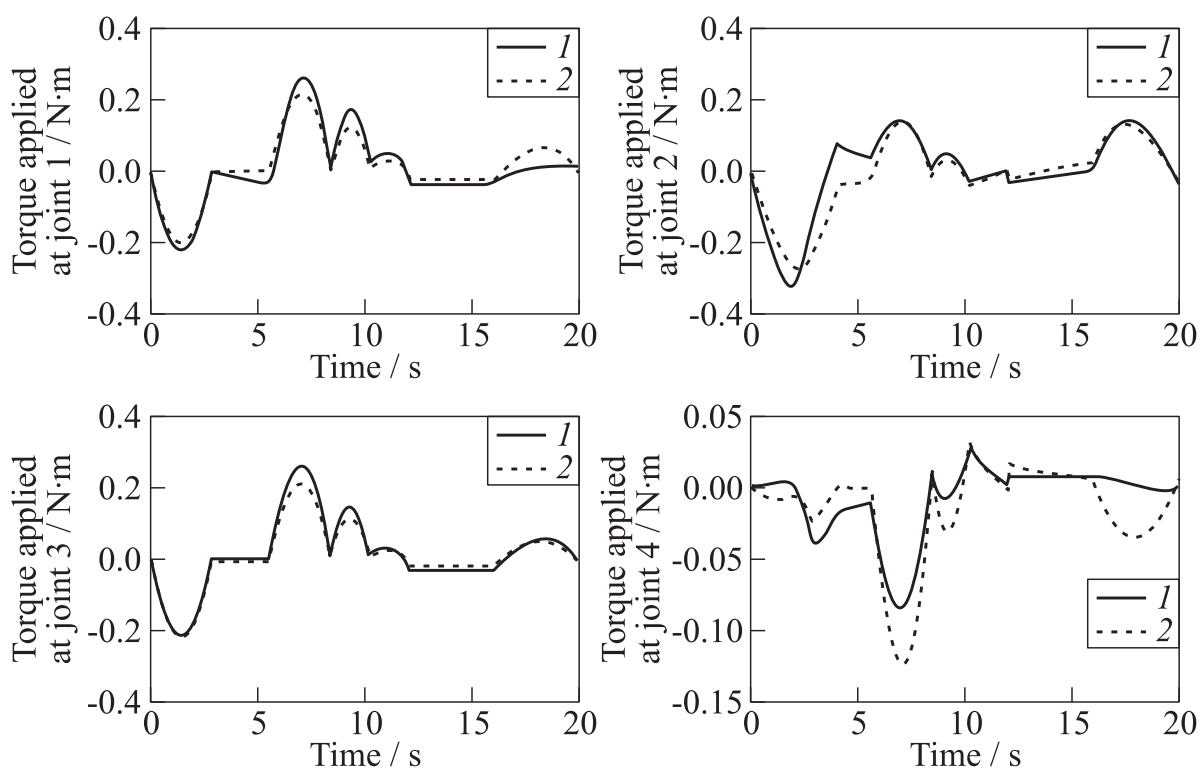

Figure 17 Torques applied at the first four manipulator joints during the capture manoeuver (comparison between the free-floating (1) and fixed-base (2) cases)

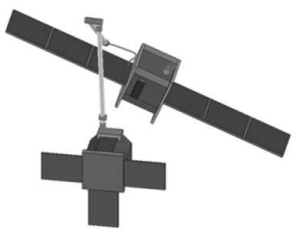

1

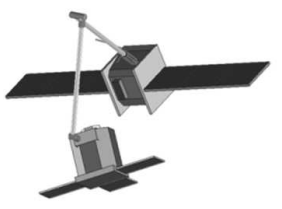

4

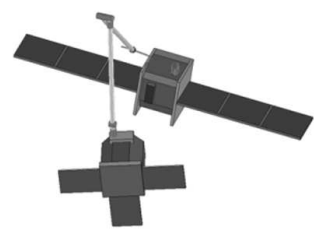

2

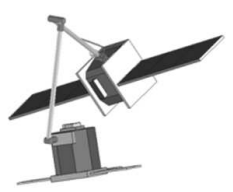

5

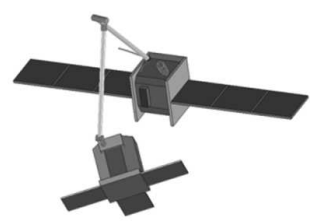

3

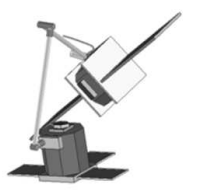

6

Figure 18 Frames from the animation presenting the capture manoeuvre performed by the free-floating satellite 


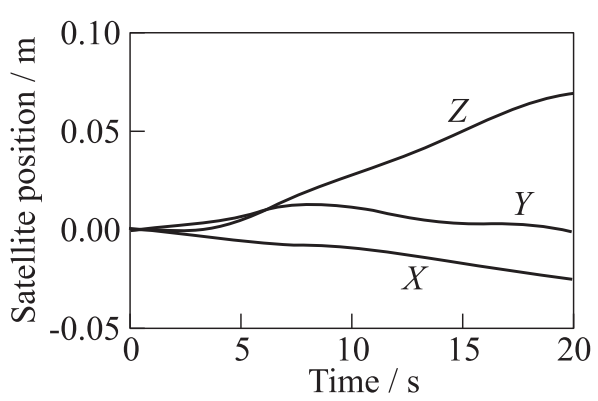

(a)

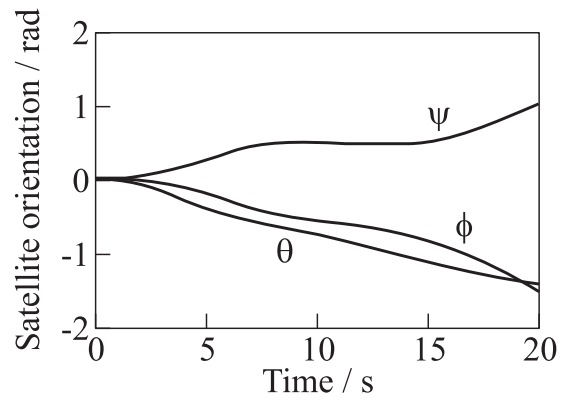

(b)

Figure 19 Position $(a)$ and orientation (b) of the manipulator-equipped satellite (free-floating case)

EE position to the final position which coincide with a position of the Docking port (DP) selected as a grasping point. Two phases of the trajectory are clearly visible in Fig. $15 b$ (second phase begins at $t=12 \mathrm{~s}$ ). In the second phase, the EE is moving on a straight-line trajectory (defined in the coordinate system that is placed at the grasping point and is rotating with the target object). The same EE trajectory is realized both for the free-floating and fixed-base cases.

To compute the velocities of manipulator joints for the free-floating case, the equations presented in section 2 are used, while for the case with the fixed satellite, corresponding equations for a standard fixed-base manipulator are used. The positions of the first four manipulator joints during the capture manoeuver for both cases are shown in Fig. 16, while torques (computed with Eq. (5)) applied at these joints are presented in Fig. 17. Frames from animation presenting the capture manoeuvre for free-floating manipulator-equipped satellite are presented in Fig. 18. Position and orientation of this satellite during the capture manoeuvre is shown in Fig. 19. For the satellite manipulator-system with parameters presented in Table 2, the influence of the manipulator motion on the state of the satellite is very significant.

\section{DISCUSSION}

Techniques for the optimization of manipulator trajectory are of special interest. The approach presented in subsection 3.2 and described in details in [6] is one of many proposed approaches for trajectory optimization (see, e.g., [17]). Application of the optimization method for a real planar system with the 2-DoF manipulator allowed for substantial reduction of a quadratic norm connected with the power use of the manipulator motors. Also, the maximal values of 
velocity and angular velocity of the satellite were lower during the realization of the optimal trajectory than during the realization of the straight-line trajectory. The optimization techniques could be utilized not only to reduce power use of motors in manipulator joints, but also to minimize manipulator disturbances on the servicing satellite (see, e.g., [18]). However, in some cases, long computational time required for optimal trajectory planning may prevent the use of such methods.

Among many other techniques for trajectory planning in the Cartesian coordinates, the use of parametric curves (such as cubic Bézier curves) as manipulator trajectories seems advantageous [8]. The shape of the curve can be conveniently adjusted by modification of curve parameters and trajectories that avoid singularities can be found. In case of Bézier curves, selection of parameters responsible for curve shape is intuitive and simple. Moreover, algorithms can be used to make the selection of Bézier curve parameters automatic. Such approach is not computationally demanding even for $3 \mathrm{D}$ cases. The application of trajectory based on the Bézier curve was successfully demonstrated in subsection 5.2. Manipulability measure for such trajectory was sufficiently high for inverting body dynamic Jacobian in every point among the trajectory while at some point of the straight line trajectory, a configuration close to singular was reached and body dynamic Jacobian could not be inverted.

The rotational motion of the target object must be taken into account when planning EE trajectory for capturing a selected grasping point on such object. The two-phase trajectory planning with straight-line trajectory in the second phase (defined in the coordinate system fixed to the grasping point) was used as a basic approach. The same EE trajectory defined in the Cartesian space (in the inertial reference frame) can be used for free-floating or fixed-base case, i. e., for the case without active AOCS and for the case with active AOCS that can compensate for any motion of the manipulator. The obtained trajectories in the configuration space differ substantially, while control torques applied at manipulator joints are comparable. At the stage of trajectory planning, it must be known whether AOCS will be operating. A case of active AOCS that is insufficient to fully compensate reaction forces and torques induced by the motions of the manipulator might be especially problematic and should be avoided. The capture maneuver performed in the free-floating case resulted in a significant change of manipulator-equipped satellite orientation. The EE trajectories during the capture maneuver could also be optimized in order to, e.g., minimize influence of the manipulator motion on the satellite.

\section{CONCLUDING REMARKS}

In this paper, the following approaches to EE trajectory planning have been reviewed: 
(i) trajectory optimization that allows minimization of a quadratic norm connected with the power use of motors;

(ii) application of the Bézier curves for computationally inexpensive planning of trajectories that can be used to avoid singularities; and

(iii) two-phase trajectory planning for grasping a tumbling target object (in the second phase, a straight-line trajectory defined in the coordinate system fixed to the grasping point is used).

Two tools for analysis and validation of trajectory planning algorithms were presented:

(i) planar air-bearing microgravity simulator which allows experiments with free-floating satellite-manipulator system in two dimensions; and

(ii) simulation tool for space robotics which is used for numerical simulations of a satellite with 7-DoF redundant manipulator.

Realization of the optimal trajectory was successfully demonstrated using the planar air-bearing microgravity simulator. Such experiments play an important role in development of any space-related technologies, as through demonstration they allow rising of the Technology Readiness Level. Moreover, the results obtained during such experiments are used for validation of the numerical simulation tool. Application of the Bézier curves for singularity avoidance and two-phase trajectories for capturing grasping point on the tumbling target object was demonstrated with the presented simulation tool for space robotics. Differences between the case with active AOCS that keeps constant position and orientation of the satellite and free-floating case were shown in the capture manoeuvre.

\section{ACKNOWLEDGMENTS}

This paper was partially supported by The National Centre for Research and Development (projects Nos. LIDER/10/89/L-2/10/NCBIR/2011 and PBS3/A3/ $22 / 2015$ ).

\section{REFERENCES}

1. Ellery, A., J. Kreisel, and B. Sommer. 2008. The case for robotic on-orbit servicing of spacecraft: Spacecraft reliability is a myth. Acta Astronaut. 63:632-648. 
2. Liou, J.-C., N. L. Johnson, and N. M. Hill. 2010. Controlling the growth of future LEO debris populations with active debris removal. Acta Astronaut. 66(5-6):648653.

3. Jaekel, S., R. Lampariello, G. Panin, et al. 2015. Robotic capture and de-orbit of a heavy, uncooperative and tumbling target satellite in low Earth orbit. 13th Symposium on Advanced Space Technologies in Robotics and Automation Proceedings. Noordwijk, The Netherlands.

4. Dubowsky, S., and E. Papadopoulos. 1993. The kinematics, dynamics, and control of free-flying and free-floating space robotic systems. IEEE T. Robotic. Autom. 9(5):531-543.

5. Rybus, T., J. Lisowski, K. Seweryn, and T. Barciński. 2012. Numerical simulations and analytical analyses of the orbital capture manoeuvre as a part of the manipulator-equipped servicing satellite design. 17th Conference (International) on Methods and Models in Automation and Control Proceedings. Miedzyzdroje, Poland.

6. Seweryn, K., and M. Banaszkiewicz. 2008. Optimization of the trajectory of a general free-flying manipulator during the rendezvous maneuver. AIAA Guidance, Navigation, and Control Conference and Exhibit Proceedings. Honolulu, HI.

7. Tsai, L.-W. 1999. Robot analysis: The mechanics of serial and parallel manipulators. John Wiley \& Sons. 520 p.

8. Rybus, T., T. Barciński, J. Lisowski, et al. 2013. Experimental demonstration of singularity avoidance with trajectories based on the Bézier curves for free-floating manipulator. 9th Workshop (International) on Robot Motion and Control Proceedings. Wąsowo, Poland.

9. Li, C., B. Liang, and W. Xu. 2006. Autonomous trajectory planning of free-floating robot for capturing space target. IEEE/RSJ Conference (International) on Intelligent Robots and Systems Proceedings. Beijing, China.

10. Rybus, T., K. Seweryn, M. Banaszkiewicz, et al. 2012. Dynamic simulations of free-floating space robots. Robot motion and control 2011. Ed. K. R. Kozłowski. Lecture notes in control and information sciences. Springer-Verlag. 422:351-361.

11. Rybus, T., and K. Seweryn. 2015. Application of rapidly-exploring random trees (RRT) algorithm for trajectory planning of free-floating space manipulator. 10th Workshop (International) on Robot Motion and Control Proceedings. Poznań, Poland.

12. Rybus, T., T. Barciński, J. Lisowski, et al. 2013. New planar air-bearing microgravity simulator for verification of space robotics numerical simulations and control algorithms. 12th Symposium on Advanced Space Technologies in Robotics and Automation Proceedings. Noordwijk, The Netherlands.

13. Rybus, T., and K. Seweryn. 2013. Trajectory planning and simulations of the manipulator mounted on a free-floating satellite. Aerospace robotics. GeoPlanet: Earth and planetary sciences book ser. Ed. J. Z. Sassiadek. Springer-Verlag. 61-73.

14. Haug, E. 1989. Computer aided kinematics and dynamics of mechanical systems. Vol. 1: Basic methods. Allyn and Bacon ser. in engineering. Boston-LondonSydney-Toronto: Allyn and Bacon. 500 p. 
15. Wood, G., and D. Kennedy. 2003. Simulating mechanical systems in Simulink with SimMechanics. Natick, USA: The MathWorks, Inc. Technical Report.

16. Seweryn, K., T. Rybus, J. Lisowski, et al. 2014. The laboratory model of the manipulator arm (WMS1 LEMUR) dedicated for on-orbit operation. 12th Symposium (International) on Artificial Intelligence, Robotics and Automation in Space Proceedings. Saint-Hubert, Quebec, Canada.

17. Flores-Abad, A., Z. Wei, O. Ma, and K. Pham. 2014. Optimal control of space robots for capturing a tumbling object with uncertainties. J. Guid. Control Dynam. 37(6):2014-2017.

18. Kaigom, E. G., T. J. Jung, and J. Rossmann. 2011. Optimal motion planning of a space robot with base disturbance minimization. 11th Symposium on Advanced Space Technologies in Robotics and Automation Proceedings. Noordwijk, The Netherlands. 